\title{
Workload and Injury in Professional Soccer Players: Role of Injury Tissue Type and Injury Severity
}

\author{
Authors \\ Kevin Enright ${ }^{1}$, Matthew Green ${ }^{3}$, Gordon Hay ${ }^{4}$, James J. Malone ${ }^{2}$
}

\section{Affiliations}

1 Research Institute for Sport and Exercise Sciences, Liverpool John Moores University, Tom Reilly Building, Byrom St Campus, Liverpool L3 3AF, UK

2 School of Health Sciences, Liverpool Hope University, Liverpool, United Kingdom of Great Britain and Northern Ireland

3 Football Association Premier League, The Premier League, London, United Kingdom of Great Britain and Northern Ireland

4 Public Health Institute, Liverpool John Moores University, Liverpool, United Kingdom of Great Britain and Northern Ireland

Key words

football, training, ACWR, load, GPS

\section{Bibliography}

DOI https://doi.org/10.1055/a-0997-6741

Published online: 4.12.2019

Int J Sports Med 2020; 41: 89-97

(c) Georg Thieme Verlag KG Stuttgart · New York

ISSN 0172-4622

\section{Correspondence}

Dr. Kevin Enright, PhD

School of Sport Studies, Leisure and Nutrition

Liverpool John Moores University

Barkhill Rd, Aigburth

L17 6BD Liverpool

United Kingdom of Great Britain and Northern Ireland

Tel.: + 151/231/5 205, Fax: + 151/231/5 205

k.j.enright@ljmu.ac.uk

\section{ABSTRACT}

The purpose of the present study was to examine the influence of workload prior to injury on injury (tissue type and severity) in professional soccer players. Twenty-eight days of retrospective training data prior to non-contact injuries $(n=264)$ were collated from 192 professional soccer players. Each injury tissue type (muscle, tendon and ligament) and severity (days missed) were categorised by medical staff. Training data were recorded using global positioning system (GPS) devices for total distance (TD), high speed distance (HSD, $>5.5 \mathrm{~m} / \mathrm{s}^{-1}$ ), and sprint distance (SPR, > $\left.7.0 \mathrm{~m} / \mathrm{s}^{-1}\right)$. Accumulated 1, 2, 3, 4-weekly loads and acute:chronic workload ratios (ACWR) (coupled, uncoupled and exponentially weighted moving average (EWMA) approaches) were calculated. Workload variables and injury tissue type were compared using a one-way ANOVA. The association between workload variables and injury severity were examined using a bivariate correlation. There were no differences in accumulated weekly loads and ACWR calculations between muscle, ligament, and tendon injuries $(P>0.05)$. Correlations between each workload variable and injury severity highlighted no significant associations $(P>0.05)$. The present findings suggest that the ability of accumulated weekly workload or ACWR methods to differentiate between injury type and injury severity are limited using the present variables.

\section{Introduction}

Soccer is a complex contact sport with high physical, technical, and tactical demands at the elite level [1]. Barnes et al. [2] highlighted the ever-increasing high-intensity demands of professional soccer in the modern game, with an increase in sprint distance of 35\% over a 7-season period. Because of the intense physical nature of the sport, a high level of injuries has been reported across a range of professional clubs [3]. In particular, non-contact muscular injuries appear to be a significant issue for both coaching and medical staff, accounting for almost one third of all time-loss injuries in men's professional soccer $[4,5]$. Financially, the average cost of a first-team player in a professional team being injured for 1 month is calculated to be worth around $€ 500000$ [6]. Despite the increased body of knowledge and applied injury prevention strategies around non-contact injuries within soccer, the rate of these types of injuries continues to rise [7].

Within professional soccer, it is commonplace for sport science staff to monitor a range of variables across the training programme [8]. The monitoring of training load (TL) on a daily basis is now standard practice in order to help facilitate the prescription of the 
correct 'dose' of TL to maximise adaptation and minimise injury risk. Measures of TL can be categorized into either external (i. e., exercise prescription by the coach) or internal (i.e., physiological stress imposed on the players) [9]. The evolution in the accessibility of wearable technology within soccer has led to the widespread use of global positioning systems (GPS) to quantify athlete movements during training and match play [8]. Common measures collected and monitored in elite soccer include high-speed distance covered $\left(>5.5 \mathrm{~m} / \mathrm{s}^{-1}\right)$, acceleration/deceleration efforts, and estimated metabolic power [8]. Sports science and medical practitioners can subsequently create individualised monitoring strategies based on the GPS data to feed back information to ensure observed $\mathrm{TL}$ is compliant with the training planned by the coaches.

Elite-level soccer players typically sustain two injuries per season, resulting in 50 injuries within a squad of 25 players [4]. It has been previously suggested that the incorrect application of workload can act as an external risk factor for injury in athletes [10]. In particular, a sudden increase in the TL placed upon an athlete (i. e., 'spike') [11] or insufficient chronic TL stimulus [12] can contribute to an increased injury risk in athletes. There has been growing use of the acute:chronic workload ratio (ACWR) in order to monitor and prescribe appropriate TLs in athletes [13]. The calculation involves the assessment of the current 1-week workload (acute) relative to the chronic workload (typically 2, 3, or 4-weekly average) [5]. Previous research has used a combination of ACWR and/or accumulated weekly TLs to investigate the relationship with injury across a range of sports, including: rugby [14-19], Australian rules football (AFL) [20-26], American football [27, 28], handball [29], Gaelic football [30], and soccer [12,31-37]. Despite this growing body of research, there have been conflicting findings within the literature. One of the reasons may be due to the range of ways in which the ACWR can be calculated. Lolli et al. [38] argue the rolling average ACWR calculation can produce spurious correlations, which can be explained by mathematical coupling, whereas others suggest that calculating the ACWR using exponentially weighted moving averages (EWMA) could provide a more sensitive model to inform decision-making [22]. To avoid error associated with ratios, researchers have also compared the cumulative totals for each variable [35]. To the authors' knowledge, few studies have calculated and compared all of the above approaches using the same training data $[22,39]$.

Within soccer, each type of non-contact injury has its own unique incidence rate and severity [40]. For example, anterior cruciate ligament injuries typically occur once every $10000 \mathrm{~h}$ of training and cause a player to be withdrawn from training for around 200 days [41]. Muscle injuries happen more often ( 1 per $1000 \mathrm{~h}$ ) and cause the player to be removed from training and competition for around 24 days [40]. Previous studies investigating the TL preceding injury have combined all non-contact injuries without distinguishing between the tissue type (e. g., tendon) and the influence of injury severity. Collating training data for each type of injury might improve our understanding of why players sustain particular injuries. If, for example, the ratio of sprinting is different prior to muscle injuries when compared to tendon or ligament injuries, this could help inform our understanding of how the musculoskeletal system responds to the training currently employed by professional soccer teams. This could also inform the decision- making processes which assist how we prescribe training and implement risk management plans to reduce injury.

Therefore, the purpose of the present study was to examine the relationships of accumulated workloads, the ACWR using different methods, and injury occurrence (severity and tissue type) in a large cohort of professional soccer players.

\section{Materials and Methods}

\section{Participants}

Data were collected from professional soccer players $(n=192)$ from 8 teams competing in recognised Union of European Football Associations (UEFA) leagues. Twenty-eight days of retrospective training and injury data were collected across both the 2015/2016 and 2016/2017 seasons. All clubs and players provided written informed consent to participate in the study, which was approved by the Institutional Review Board (IRB) at Liverpool John Moores University (United Kingdom) and conformed to the recommendations of the Declaration of Helsinki and those outlined by Harriss and colleagues [42]. Goalkeepers were excluded from the study due to the different nature of their playing activity.

\section{Quantifying workload}

Training load was quantified using GPS data collected from all onpitch training sessions and matches during the in-season phase (Optimeye S5, firmware version 717, Catapult Sports, Melbourne, Australia). Each player was assigned their own specific device in order to avoid potential inter-unit reliability error [43]. The device was worn inside a custom-made vest supplied by the manufacturer that was positioned across the scapula of the players. The number of satellites and horizontal dilution of position (HDOP) across all data collections were $14.0 \pm 2$ and $0.77 \pm 0.03$, respectively. The Catapult S5 GPS device has previously shown acceptable levels of both reliability [44] and validity [45] for velocity-based variables. The data collection procedures followed the guidelines for using GPS data in sport [43]. Following each session, data were downloaded by a member of each sports science team using the manufacturer's software (Openfield, version 1.14, Catapult Sports, Melbourne, Australia). The following variables were included for data analysis: total distance (TD), high- speed distance (HSD, $\left.>5.5 \mathrm{~m} / \mathrm{s}^{-1}\right)$, and sprint distance $\left(\mathrm{SPR},>7.0 \mathrm{~m} / \mathrm{s}^{-1}\right)$. The minimum effort duration for velocity-based variables was set at 0.4 secs in line with previous recommendations [46].

\section{Injury quantification}

Injury information was recorded using the clubs' standardised internal medical procedures and were guided by the Munich Consensus statement [47]. Non-contact injury was defined as an injury that involved no physical contact from another player and resulted in absence from participation in training with the normal group of players. Within each club, medical doctors and qualified physiotherapists diagnosed and recorded each injury tissue type (muscle, tendon, or ligament) confirmed using ultrasound technology [47]. Only injuries that were sustained for the first time were included in the final analysis. As such, data for subsequent recurring injuries were excluded. The severity of each injury was quantified as the number of days missed from training with the main group of 'starting' players, involving full 
intensity and contact. The severity of each injury was also classified as either minimal ( $1-3$ days missed), mild (4-7 days missed), moderate (8-28 days missed), or severe ( $>29$ days missed) [32]. All injury data were stored in a central database and then sent to the researchers via an encrypted platform.

\section{Data analyses}

Data were categorised into 7-day blocks (weeks) starting with the most recent day to the injury occurrence regardless of the week day. Accumulated 1, 2, 3, 4-weekly loads were subsequently calculated using the sum of the daily load across the previous week(s). ACWRs were calculated using the GPS-derived data collected across the 28-day period prior to each injury. The last session recorded before the injury was classified as 'day 1 '. From this day, the data were categorised into 7-day phases using a rolling average approach prior to this point (regardless of the day of the week). The acute training load was defined as the average 'load' for the 7 days prior to the injury. Both 'coupled' (C) and 'uncoupled' (UC) ACWRs were calculated [52]. As a result, the chronic aspect of the ratio included either: a) the average of the $2^{\text {nd }}$ and $3^{\text {rd }}$ week prior to the injury (UC ACWR 1:3); b) the $2^{\text {nd }}, 3^{\text {rd }}$, and $4^{\text {th }}$ week prior to injury (UC ACWR $1: 4)$; c) the average of the $1^{\text {st }}, 2^{\text {nd }}$ and $3^{\text {rd }}$ week prior to the injury (C ACWR 1:3); or d) the $1^{\text {st }}, 2^{\text {nd }}, 3^{\text {rd }}$, and $4^{\text {th }}$ week prior to injury (C ACWR 1:4). In addition, the exponentially weighted ACWR was calculated according to the equation outlined by Williams and colleagues [48].

\section{Statistical analysis}

The software package SPSS (Version 24.0 SPSS Inc. Chicago, IL, USA) was used to conduct the statistical analysis. Prior to statistical comparisons, assessments for normality and variance assurance were made. A one-way analysis of variance (ANOVA) was subsequently used to determine whether there are any statistically significant differences between the means of each injury tissue type (muscle, tendon, and ligament) and each accumulated weekly load, coupled, uncoupled, EWMA (1:3 and 1:4), for TD, HSD and SPR. To examine the relationship between ACWR method and weekly accumulated workload on injury severity, correlations were performed using a bivariate analysis. The level of significance was set at $\mathrm{P}<0.05$. Confidence intervals $(95 \% \mathrm{Cl})$ are provided alongside descriptive data (mean \pm standard deviation (SD)).

\section{Results}

Two hundred and sixty-four non-contact injuries from eight professional teams were collected. One hundred and forty injury data sets were excluded due to inconsistent and/or missing data. Therefore, 124 lower limb injuries were included in the final analysis (muscle, $n=79$; tendon, $n=28$; ligament, $n=17$ ). Descriptive data for each injury is presented in $>$ Table 1.

\section{Influence of ACWR on injury tissue type and severity}

Workload data for each ACWR method in relation to injury tissue type and severity are presented in $>$ Table 2 . Regardless of the ACWR method used, there was no significant difference shown between injury tissue type for all workload variables $(P>0.05)$. In addition, there was no relationship found between ACWR methods and injury severity $(P>0.05)$.

\section{Influence of accumulated weekly workload on injury tissue type and severity}

Workload data for the different accumulated weekly loads in relation to injury tissue type and severity are presented in > Table 3. There was no significant relationship found across the different accumulated weekly workload calculations (1, 2, 3, and 4-weekly loads) and injury tissue type for all workload variables $(P>0.05)$. In addition, there was no relationship found between accumulated workloads and injury severity $(P>0.05)$.

\section{Discussion}

The purpose of the present study was to examine the relationships of accumulated workloads, the ACWR using different methods, and injury occurrence (severity and tissue type) in a large cohort of professional soccer players. Regardless of the ACWR method used or weekly accumulated workloads, there was no observed differences in workload variables and each injury tissue type. In addition, there was no relationship found between workload variables and injury severity. The present findings suggest that workload data typically used by professional soccer teams may not be able to discriminate between injury type and/or severity.

The relationship between the ACWR and injury risk in soccer has been previously examined in the literature [12, 30-32, 34-36, 49]. However, limited attention has been given to the ability of the ACWR to differentiate between different tissue types within noncontact injuries. Understanding if the different workloads associated with the training programme could result in each type of injury and might have practical relevance for coaches who aim to minimise the injury burden within their team. The present study highlighted that the workload exposure across both ACWR methods and accumulated weekly loads were not different before either a muscle, tendon, or a ligament injury. Considering that muscles, tendons, and ligaments have unique mechanical intensity thresholds that initiate distinct temporal responses [50], it is logical to suggest that each injury could have its specific loading pattern prior to the injury [51]. Indeed, previous research has noted that an acute

- Table 1 Descriptive information for injury incidence across all clubs.

\begin{tabular}{|l|c|c|c|c|c|c|}
\hline & \multicolumn{4}{|c|}{ Injury severity } & \multicolumn{2}{c|}{ Injury environment } \\
\cline { 2 - 7 } & $\mathbf{1 - 3} \mathbf{d}$ Minimal & $\mathbf{4 - 7} \mathbf{d}$ Mild & $\mathbf{8 - 2 8} \mathbf{d}$ Moderate & >29 d Severe & Match & Training \\
\hline Muscle & 18 & 18 & 33 & 10 & 17 & 0 \\
\hline Ligament & 0 & 0 & 0 & 4 & 17 & 10 \\
\hline Tendon & 4 & 9 & 11 & 4 & 18 \\
\hline
\end{tabular}


- Table 2 EWMA, coupled and uncoupled ACWR data for muscle, ligament, and tendon injures.

\begin{tabular}{|c|c|c|c|c|c|c|c|c|c|c|c|}
\hline \multirow[b]{3}{*}{ EWMA ACWR TD } & \multirow{3}{*}{ Mean \pm SD } & \multirow{2}{*}{\multicolumn{2}{|c|}{$\begin{array}{l}\text { 95\% Confidence Interval } \\
\text { (lower - upper) }\end{array}$}} & \multirow{3}{*}{ Min } & \multirow{3}{*}{ Max } & \multirow{3}{*}{ Range } & \multicolumn{2}{|c|}{ One-Way ANOVA } & \multirow{3}{*}{$\begin{array}{l}\text { Effect } \\
\text { Size }\left(\eta^{2}\right)\end{array}$} & \multicolumn{2}{|c|}{ Correlation } \\
\hline & & & & & & & \multirow[t]{2}{*}{$\mathbf{F}$} & \multirow[t]{2}{*}{$\mathbf{P}$} & & \multirow[t]{2}{*}{ Pearson } & \multirow[t]{2}{*}{ Sig. } \\
\hline & & & & & & & & & & & \\
\hline Muscle & $1.03 \pm 0.27$ & 0.96 & 1.09 & 0.13 & 1.65 & 1.52 & \multirow{3}{*}{0.43} & \multirow{3}{*}{0.663} & \multirow{3}{*}{0.007} & \multirow{3}{*}{-0.055} & \multirow{3}{*}{0.542} \\
\hline Ligament & $0.95 \pm 0.33$ & 0.77 & 1.12 & 0.53 & 1.88 & 1.35 & & & & & \\
\hline Tendon & $1.01 \pm 0.24$ & 0.91 & 1.10 & 0.56 & 1.60 & 1.04 & & & & & \\
\hline EWMA AC & & & & & & & & & & & \\
\hline Muscle & $0.95 \pm 0.29$ & 0.88 & 1.01 & 0.12 & 1.66 & 1.55 & & & & & \\
\hline Ligament & $1.00 \pm 0.39$ & 0.79 & 1.21 & 0.42 & 1.75 & 1.32 & 0.17 & 0.898 & 0.002 & 0.031 & 0.732 \\
\hline Tendon & $0.99 \pm 0.36$ & 0.85 & 1.13 & 0.55 & 2.16 & 1.61 & & & & & \\
\hline EWMA AC & & & & & & & & & & & \\
\hline Muscle & $0.93 \pm 0.42$ & 0.83 & 1.03 & 0.12 & 2.07 & 1.95 & & & & & \\
\hline Ligament & $0.99 \pm 0.57$ & 0.68 & 1.29 & 0.12 & 1.98 & 1.86 & 0.09 & 0.924 & 0.001 & 0.013 & 0.888 \\
\hline Tendon & $0.98 \pm 0.51$ & 0.78 & 1.18 & 0.08 & 2.22 & 2.14 & & & & & \\
\hline 1:4 ACWF & & & & & & & & & & & \\
\hline Muscle & $1.06 \pm 0.32$ & 0.99 & 1.14 & 0.20 & 1.96 & 1.76 & & & & & \\
\hline Ligament & $1.04 \pm 0.35$ & 0.85 & 1.22 & 0.59 & 2.23 & 1.64 & 0.2 & 0.819 & 0.003 & -0.016 & 0.861 \\
\hline Tendon & $1.03 \pm 0.36$ & 0.89 & 1.18 & 0.36 & 2.18 & 1.82 & & & & & \\
\hline 1:4 ACWF & & & & & & & & & & & \\
\hline Muscle & $0.99 \pm 0.39$ & 0.90 & 1.09 & 0.02 & 2.26 & 2.23 & & & & & \\
\hline Ligament & $1.08 \pm 0.30$ & 0.92 & 1.23 & 0.58 & 1.57 & 0.99 & 0.16 & 0.856 & 0.003 & 0.010 & 0.911 \\
\hline Tendon & $1.07 \pm 0.46$ & 0.89 & 1.25 & 0.23 & 2.64 & 2.41 & & & & & \\
\hline 1:4 ACWF & & & & & & & & & & & \\
\hline Muscle & $1.07 \pm 0.66$ & 0.91 & 1.22 & 0.00 & 2.87 & 2.87 & & & & & \\
\hline Ligament & $1.14 \pm 0.66$ & 0.79 & 1.49 & 0.12 & 2.72 & 2.59 & 0.38 & 0.721 & 0.005 & 0.038 & 0.678 \\
\hline Tendon & $1.01 \pm 0.61$ & 0.77 & 1.25 & 0.00 & 2.64 & 2.64 & & & & & \\
\hline 1:3 ACWF & & & & & & & & & & & \\
\hline Muscle & $1.06 \pm 0.30$ & 0.99 & 1.13 & 0.23 & 2.12 & 1.89 & & & & & \\
\hline Ligament & $1.07 \pm 0.24$ & 0.94 & 1.19 & 0.60 & 1.69 & 1.08 & 0.52 & 0.596 & 0.009 & 0.006 & 0.943 \\
\hline Tendon & $1.00 \pm 0.28$ & 0.89 & 1.11 & 0.38 & 1.92 & 1.54 & & & & & \\
\hline 1:3 ACWF & & & & & & & & & & & \\
\hline Muscle & $0.99 \pm 0.37$ & 0.91 & 1.08 & 0.03 & 1.96 & 1.93 & & & & & \\
\hline Ligament & $1.09 \pm 0.23$ & 0.96 & 1.21 & 0.72 & 1.55 & 0.83 & 0.12 & 0.894 & 0.002 & 0.014 & 0.877 \\
\hline Tendon & $1.04 \pm 0.37$ & 0.89 & 1.18 & 0.27 & 2.31 & 2.04 & & & & & \\
\hline 1:3 ACWF & & & & & & & & & & & \\
\hline Muscle & $1.06 \pm 0.62$ & 0.92 & 1.20 & 0.00 & 2.66 & 2.66 & & & & & \\
\hline Ligament & $1.13 \pm 0.55$ & 0.84 & 1.42 & 0.22 & 2.06 & 1.84 & 0.64 & 0.511 & 0.011 & 0.034 & 0.710 \\
\hline Tendon & $0.96 \pm 0.51$ & 0.75 & 1.16 & 0.00 & 2.37 & 2.37 & & & & & \\
\hline 1:4 ACWF & & & & & & & & & & & \\
\hline Muscle & $0.50 \pm 0.25$ & 0.45 & 0.56 & 0.06 & 1.83 & 1.77 & & & & & \\
\hline Ligament & $0.53 \pm 0.51$ & 0.26 & 0.80 & 0.22 & 2.38 & 2.16 & 0.17 & 0.898 & 0.005 & -0.157 & 0.082 \\
\hline Tendon & $0.52 \pm 0.30$ & 0.40 & 0.63 & 0.13 & 1.36 & 1.23 & & & & & \\
\hline 1:4 ACWF & & & & & & & & & & & \\
\hline Muscle & $0.48 \pm 0.30$ & 0.41 & 0.55 & 0.01 & 1.85 & 1.85 & & & & & \\
\hline Ligament & $0.55 \pm 0.33$ & 0.37 & 0.72 & 0.20 & 1.51 & 1.31 & 0.78 & 0.452 & 0.013 & -0.032 & 0.729 \\
\hline Tendon & $0.57 \pm 0.48$ & 0.38 & 0.75 & 0.08 & 2.27 & 2.20 & & & & & \\
\hline 1:4 ACWF & & & & & & & & & & & \\
\hline Muscle & $0.63 \pm 0.66$ & 0.47 & 0.78 & 0.00 & 3.66 & 3.66 & & & & & \\
\hline Ligament & $0.79 \pm 1.08$ & 0.22 & 1.37 & 0.03 & 4.56 & 4.52 & 0.56 & 0.604 & 0.008 & -0.051 & 0.576 \\
\hline Tendon & $0.58 \pm 0.50$ & 0.38 & 0.77 & 0.00 & 1.95 & 1.95 & & & & & \\
\hline
\end{tabular}


- Table 2 Continued.

\begin{tabular}{|c|c|c|c|c|c|c|c|c|c|c|c|}
\hline & \multirow{3}{*}{ Mean \pm SD } & \multirow{2}{*}{\multicolumn{2}{|c|}{$\begin{array}{l}\text { 95\% Confidence Interval } \\
\text { (lower - upper) }\end{array}$}} & \multirow{3}{*}{ Min } & \multirow{3}{*}{ Max } & \multirow{3}{*}{ Range } & \multicolumn{2}{|c|}{ One-Way ANOVA } & \multirow{3}{*}{$\begin{array}{l}\text { Effect } \\
\text { Size }\left(\eta^{2}\right)\end{array}$} & \multicolumn{2}{|c|}{ Correlation } \\
\hline & & & & & & & $\mathbf{F}$ & $\mathbf{P}$ & & Pearson & Sig. \\
\hline \multicolumn{7}{|c|}{ 1:3 ACWR [UC] TD } & & & & & \\
\hline Muscle & $0.89 \pm 0.42$ & 0.79 & 0.98 & 0.10 & 2.32 & 2.22 & \multirow{3}{*}{0.06} & \multirow{3}{*}{0.942} & \multirow{3}{*}{0.001} & \multirow{3}{*}{-0.101} & \multirow{3}{*}{0.262} \\
\hline Ligament & $0.86 \pm 0.45$ & 0.62 & 1.10 & 0.37 & 2.38 & 2.01 & & & & & \\
\hline Tendon & $0.86 \pm 0.51$ & 0.65 & 1.06 & 0.20 & 2.79 & 2.58 & & & & & \\
\hline \multicolumn{12}{|c|}{ 1:3 ACWR [UC] HSD } \\
\hline Muscle & $0.84 \pm 0.46$ & 0.74 & 0.95 & 0.01 & 2.13 & 2.12 & \multirow{3}{*}{0.14} & \multirow{3}{*}{0.901} & \multirow{3}{*}{0.002} & \multirow{3}{*}{-0.049} & \multirow{3}{*}{0.596} \\
\hline Ligament & $0.95 \pm 0.41$ & 0.73 & 1.17 & 0.38 & 1.80 & 1.42 & & & & & \\
\hline Tendon & $0.96 \pm 0.84$ & 0.63 & 1.29 & 0.14 & 4.16 & 4.02 & & & & & \\
\hline \multicolumn{12}{|c|}{ 1:3 ACWR [UC] SPR } \\
\hline Muscle & $1.26 \pm 1.46$ & 0.93 & 1.60 & 0.00 & 7.67 & 7.67 & \multirow{3}{*}{0.63} & \multirow{3}{*}{0.522} & \multirow{3}{*}{0.011} & \multirow{3}{*}{-0.044} & \multirow{3}{*}{0.640} \\
\hline Ligament & $1.21 \pm 1.07$ & 0.65 & 1.78 & 0.10 & 4.56 & 4.46 & & & & & \\
\hline Tendon & $0.94 \pm 0.75$ & 0.64 & 1.23 & 0.00 & 3.58 & 3.58 & & & & & \\
\hline
\end{tabular}

Abbreviations: EWMA, exponentially weighted moving average; ACWR, acute chronic ratio; TD, total distance; HSD, high-speed distance; SPR, sprint distance; C, coupled; UC, uncoupled; ACC, accumulative; $\eta^{2}$, eta squared.

increase in sprinting is associated with muscle-based injuries [12]. This is supported by experimental research which demonstrates the transfer of force from ground to bone, from bone to tendon, and from tendon to muscle is higher during sprinting actions [52]. It was anticipated that muscle injuries would have occurred in individuals who underwent a 'spike' in sprint-based activity in the week before the injury [31,32]. However, our results highlight that the training data for each player is homogeneous regardless of the type of injury. We also observed no differences in the ACWR (i.e., coupled, uncoupled or EWMA) for each of the workload measures included in this study (total distance, high speed distance, and sprint distance) across each injury tissue type. This suggests that the exposure to use of the ACWR and accumulated weekly loads may not be sensitive enough to detect differences in non-contact injury tissue types in professional soccer players.

It is possible that the lack of differences observed in ACWR between each injury tissue type could be partly explained by the workload variables examined in the present study. Soccer training and match play include an array of sport-specific skills (e. g., dribbling, passing, and shooting) interspersed with repeated explosive activities and movements (e. g., high-speed running, sprinting, jumping, and tackling) [1]. Unfortunately, such movements could not be identified by the 'distance-based' variables used in the present study. Indeed, an increased amount of jumping and landing places additional stress on tendons and may injure the vulnerable junctional zones (i.e., the myotendinous junction and the enthesis). Owing to the limited number of consistent variables returned from each club and the strict inclusion criteria in the present study, we were unable to quantify the amount of jumping and landing. Therefore, at present it is unclear if differential training stimuli result in a unique physiological response for each tissue type, subsequently influencing the types of non-contact injury sustained by players. This still remains an important question which will require further attention in future research. To do this, investigating other TL variables that might be able to capture the 'uncontrolled nature' of soccer training is warranted. The inclusion of accelerometer data might be able to provide a more complete picture of the different degrees of mechanical load associated with different movements players experience during training and match play [51]. Indeed, considering the diverse physiological responses on bone, muscle, tendon, and ligament tissue following different intensities of exercise [50], it is possible that a more detailed description of the overall mechanical and physiological load could show differences in the training stresses prior to different types of injury [51].

Previous authors have suggested that an ACWR 'sweet spot' exists (around 0.85-1.35), which could reduce the likelihood of injury and provide a positive training stimulus to prevent injury [53]. This is supported by Colby et al. [21], who noted that players with a 'moderate' ACWR for sprint distance had a lower injury risk when compared to players who experienced 'extremely low' and 'extremely high' sprint ACWRs. This suggests that a rapid increase in sprinting within a short time period should be avoided to reduce the likelihood of muscle injuries $[11,18]$. This concept was also recently supported by Jaspers et al. [27], who note a lower injury risk was found for ACWR values between 1.00 and 1.25 in professional soccer players. The authors also noted beneficial effects for medium ACWRs, showing a decreased injury risk in the subsequent week. This is in line with earlier research in different team sports suggesting that a gradual increase of sprint-based activity over time is likely to have a preventative effect on muscle injuries [12]. These observations were, however, not supported within the current study. Conversely, almost all 142 non-contact injuries occurred within the suggested 'sweet spot' zone [53]. This highlights that injuries in the current population occur regardless of the fluctuation in the workload experienced in the weeks preceding injury. Collectively, this further underlines the complexity of risk factors associated with injury as previously highlighted by Windt and Gabbett $[10,54]$. The authors highlight both internal (e. g., current fitness status, the player's unique anatomy) and external risk factors (e. g., the playing surface or footwear/equipment used) interact and ultimately result in an inciting event. In addition, although not discussed by Windt et al., genetic predisposition [55], muscle 
- Table 3 Accumulated weekly workload data for injury tissue type and relationship with severity.

\begin{tabular}{|c|c|c|c|c|c|c|c|c|c|c|}
\hline \multirow{3}{*}{$\begin{array}{l}\text { Workload } \\
\text { Variable } \\
\text { ACC TD Wk }\end{array}$} & \multirow{3}{*}{ Mean \pm SD } & \multirow{2}{*}{\multicolumn{2}{|c|}{$\begin{array}{l}\text { 95\% Confidence Interval } \\
\text { (lower - upper) }\end{array}$}} & \multirow{3}{*}{ Min } & \multirow{3}{*}{ Max } & \multirow{3}{*}{ Range } & \multicolumn{2}{|c|}{ One-Way ANOVA } & \multicolumn{2}{|c|}{ Correlation } \\
\hline & & & & & & & \multirow[t]{2}{*}{$\mathbf{F}$} & \multirow[t]{2}{*}{$\mathbf{P}$} & \multirow[t]{2}{*}{ Pearson } & \multirow[t]{2}{*}{ Sig. } \\
\hline & & & & & & & & & & \\
\hline Muscle & $26837 \pm 8818$ & 24794 & 28880 & 4452 & 48860 & 44408 & \multirow{3}{*}{0.881} & \multirow{3}{*}{0.417} & \multirow{3}{*}{-0.065} & \multirow{3}{*}{0.474} \\
\hline Ligament & $23483 \pm 4427$ & 21124 & 25843 & 17311 & 33127 & 15817 & & & & \\
\hline Tendon & $24240 \pm 8016$ & 21069 & 27411 & 8554 & 37452 & 28898 & & & & \\
\hline \multicolumn{11}{|c|}{ ACC TD Wk 2} \\
\hline Muscle & $52124 \pm 12496$ & 49229 & 55019 & 20944 & 84692 & 63749 & \multirow{3}{*}{1.038} & \multirow{3}{*}{0.357} & & \\
\hline Ligament & $45331 \pm 9585$ & 40223 & 50438 & 26490 & 58996 & 32506 & & & -0.047 & 0.607 \\
\hline Tendon & $50727 \pm 13423$ & 45417 & 56037 & 26314 & 74802 & 48488 & & & & \\
\hline ACC TD Wk & & & & & & & & & & \\
\hline Muscle & $76320 \pm 15704$ & 72682 & 79959 & 34278 & 112768 & 78491 & & & & \\
\hline Ligament & $69165 \pm 13863$ & 61778 & 76553 & 51389 & 91024 & 39635 & 0.706 & 0.495 & -0.009 & 0.920 \\
\hline Tendon & $74395 \pm 18406$ & 67114 & 81676 & 37020 & 100297 & 63278 & & & & \\
\hline ACC TD Wk & & & & & & & & & & \\
\hline Muscle & $101072 \pm 18656$ & 96750 & 105394 & 57936 & 140670 & 82734 & & & & \\
\hline Ligament & $95071 \pm 19990$ & 84420 & 105723 & 52067 & 127476 & 75409 & 0.311 & 0.734 & 0.014 & 0.881 \\
\hline Tendon & $96559 \pm 24174$ & 86996 & 106122 & 45788 & 132093 & 86305 & & & & \\
\hline ACC HSD W & & & & & & & & & & \\
\hline Muscle & $1179 \pm 560$ & 1050 & 1309 & 31 & 2679 & 2648 & & & & \\
\hline Ligament & $1127 \pm 469$ & 878 & 1377 & 502 & 2293 & 1791 & 0.107 & 0.898 & 0 & 0.997 \\
\hline Tendon & $1139 \pm 482$ & 948 & 1330 & 330 & 1841 & 1512 & & & & \\
\hline ACC HSD W & & & & & & & & & & \\
\hline Muscle & $2431 \pm 891$ & 2225 & 2638 & 482 & 4609 & 4127 & & & & \\
\hline Ligament & $2256 \pm 1096$ & 1672 & 2840 & 1021 & 4807 & 3786 & 0.167 & 0.846 & -0.002 & 0.980 \\
\hline Tendon & $2322 \pm 891$ & 1969 & 2674 & 699 & 3993 & 3293 & & & & \\
\hline ACC HSD W & & & & & & & & & & \\
\hline Muscle & $3563 \pm 1103$ & 3308 & 3819 & 1258 & 6592 & 5334 & & & & \\
\hline Ligament & $3143 \pm 1281$ & 2461 & 3825 & 1664 & 6214 & 4550 & 0.715 & 0.491 & -0.113 & 0.211 \\
\hline Tendon & $3514 \pm 1423$ & 2951 & 4077 & 802 & 5780 & 4978 & & & & \\
\hline ACC HSD W & & & & & & & & & & \\
\hline Muscle & $4729 \pm 1346$ & 4417 & 5041 & 1842 & 7706 & 5864 & & & & \\
\hline Ligament & $4188 \pm 1516$ & 3381 & 4996 & 2110 & 7266 & 5156 & 0.816 & 0.445 & -0.061 & 0.500 \\
\hline Tendon & $4613 \pm 1975$ & 3832 & 5394 & 1294 & 7570 & 6276 & & & & \\
\hline ACC SPR W & & & & & & & & & & \\
\hline Muscle & $247 \pm 195$ & 201 & 292 & 0 & 965 & 965 & & & & \\
\hline Ligament & $246 \pm 155$ & 164 & 329 & 41 & 552 & 512 & 0.017 & 0.983 & -0.84 & 0.355 \\
\hline Tendon & $234 \pm 161$ & 170 & 297 & 0 & 743 & 743 & & & & \\
\hline ACC SPR W & & & & & & & & & & \\
\hline Muscle & $474 \pm 289$ & 407 & 541 & 2 & 1314 & 1312 & & & & \\
\hline Ligament & $512 \pm 414$ & 291 & 732 & 71 & 1437 & 1366 & 0.345 & 0.709 & -0.186 & 0.038 \\
\hline Tendon & $509 \pm 258$ & 407 & 611 & 23 & 1068 & 1045 & & & & \\
\hline ACC SPR W & & & & & & & & & & \\
\hline Muscle & $695 \pm 385$ & 606 & 784 & 43 & 1705 & 1662 & & & & \\
\hline Ligament & $707 \pm 508$ & 436 & 977 & 193 & 1757 & 1564 & 0.246 & 0.783 & -0.094 & 0.300 \\
\hline Tendon & $740 \pm 426$ & 571 & 908 & 23 & 1693 & 1670 & & & & \\
\hline ACC SPR W & & & & & & & & & & \\
\hline Muscle & $930 \pm 504$ & 813 & 1047 & 106 & 2572 & 2466 & & & & \\
\hline Ligament & $905 \pm 548$ & 613 & 1197 & 261 & 2071 & 1811 & 0.107 & 0.899 & -0.001 & 0.992 \\
\hline Tendon & $953 \pm 549$ & 736 & 1170 & 92 & 2437 & 2344 & & & & \\
\hline
\end{tabular}

Abbreviations: ACC, accumulated workload; TD, total distance; HSD, high-speed distance; SPR, sprint distance; C, coupled; UC, uncoupled; WK, number of accumulated weeks of workload data. 
soreness [56], sleep quality [57], muscle architecture [58], and other stressors associated with competing at the elite level are also likely to affect an individual's injury risk and warrant further attention.

Severe injuries remove players from match play for lengthy durations, often resulting in significant psychological distress for the athlete [59] and a reduction in the teams' performance [60] whilst also having financial implications for professional teams [6]. It is, therefore, important that we aim to understand if the severity of injury may share an association with the workload undertaken by soccer players. However, few studies conducted to date have investigated the relationship between workload and the severity of injury $[16,17,23,31,32]$. These previous studies have reported the severity of injury in one of 4 categories (minimal, mild, moderate, and severe) associated with the number of days missed from training and/or games. However, categorising the injury severity in this way limits researchers ability to study the effect of workload on injury severity. Therefore, the present study reported the absolute number of days missed from training/match play. Using this approach, our results indicated that none of the ACWRs or accumulated weekly loads for TD, HSD, or SPR distance was associated with the severity of injury. This finding suggests that workload distance-based data, although important to monitor in a practical sense, has no associative value for the number of days a player will miss following injury. Even though the present study did not find any association, it is important that future research attempts to understand how training load interacts with other individual factors such as fitness level using advanced statistical techniques [54,61]. Although appreciating cause and effect is important, understanding the mechanisms which influence the individual and the outcome are vital if we intend to reduce the injury burden currently evident within professional soccer.

\section{Conclusion}

The present study is the first to investigate non-contact injury tissue type and injury severity in professional soccer players using a range of ACWR methods and weekly accumulated workloads. Regardless of the ACWR method used or weekly accumulated workloads, there was no observed differences in workload variables and each injury tissue type. In addition, there was no relationship found between workload variables and injury severity. The current findings reinforce that distance-based workload variables (i.e., TD, HSD, SPR) may not be sensitive enough to differentiate between different injury tissue types. Therefore, the use of ACWRs in isolation should, be acknowledged as a limited approach. As the physiological and biomechanical load-adaptation pathways have diverse response rates, there appears to be a need for studies to investigate the role of different degrees of physiological and biomechanical training load on different tissue types. Moreover, considering the physiological and psychological response to each training exposure in the context of the players' current fitness level and mental condition could allow us to gain more insight into why players get injured. Findings from such research is likely to have implications for the planning of training to prevent injury.

\section{Acknowledgements}

The authors would like to thank all of the clubs who took part and provided data for the present study.

\section{Conflict of Interest}

The authors declare that they have no conflict of interest.

\section{References}

[1] Fransson D, Vigh-Larsen JF, Fatouros IG et al. Fatigue responses in various muscle groups in well-trained competitive male players after a simulated soccer game. J Hum Kinet 2018; 61: 85-97

[2] Barnes C, Archer DT, Hogg B et al. The evolution of physical and technical performance parameters in the English Premier League. Int J Sports Med 2014; 35: 1095-1100

[3] Ekstrand J, Lundqvist D, Davison M et al. Communication quality between the medical team and the head coach/manager is associated with injury burden and player availability in elite football clubs. $\mathrm{Br}$ ] Sports Med 2019; 53: 304-308

[4] Ekstrand J, Hagglund M, Walden M. Epidemiology of muscle injuries in professional football (soccer). Am J Sports Med 2011; 39: 1226-1232

[5] Jaspers A, Brink MS, Probst SGM et al. Relationships between training load indicators and training outcomes in professional soccer. Sports Med 2017; 47: 533-544

[6] Ekstrand J. Keeping your top players on the pitch: The key to football medicine at a professional level. Br J Sports Med 2013; 47: 723-724

[7] Ekstrand J, Walden M, Hagglund M. Hamstring injuries have increased by $4 \%$ annually in men's professional football since 2001: A 13-year longitudinal analysis of the UEFA Elite Club injury study. $\mathrm{Br}$ ] Sports Med 2016; 50: 731-737

[8] Akenhead R, Nassis GP. Training load and player monitoring in high-level football: Current practice and perceptions. Int J Sports Physiol Perform 2016; 11: 587-593

[9] Impellizzeri FM, Rampinini E, Marcora SM. Physiological assessment of aerobic training in soccer. J Sports Sci 2005; 23: 583-592

[10] Windt J, Gabbett TJ. How do training and competition workloads relate to injury? The workload-injury aetiology model. Br J Sports Med 2017; 51: 428-435

[11] Hulin BT, Gabbett T], Blanch P et al. Spikes in acute workload are associated with increased injury risk in elite cricket fast bowlers. $\mathrm{Br}$ J Sports Med 2014; 48: 708-712

[12] Malone S, Owen A, Mendes B et al. High-speed running and sprinting as an injury risk factor in soccer: Can well-developed physical qualities reduce the risk? J Sci Med Sport 2018; 21: 257-262

[13] Hulin BT, Gabbett T], Lawson DW et al. The acute: Chronic workload ratio predicts injury: High chronic workload may decrease injury risk in elite rugby league players. Br J Sports Med 2016; 50: 231-236

[14] Cross M], Williams S, Trewartha G et al. The influence of in-season training loads on injury risk in professional rugby union. Int J Sports Physiol Perform 2016; 11: 350-355

[15] Cummins C, Welch M, Inkster B et al. Modelling the relationships between volume, intensity and injury-risk in professional rugby league players. J Sci Med Sport 2019; 22: 653-660

[16] Gabbett T]. Influence of training and match intensity on injuries in rugby league. J Sports Sci 2004; 22: 409-417

[17] Gabbett T], Jenkins DG. Relationship between training load and injury in professional rugby league players. J Sci Med Sport 2011; 14 : 204-209 
[18] Gabbett T], Ullah S. Relationship between running loads and soft-tissue injury in elite team sports athletes. J Strength Cond Res 2012; 26: 953-960

[19] Killen NM, Gabbett TJ, Jenkins DG. Training loads and incidence of injury during the preseason in professional rugby league players. J Strength Cond Res 2010; 24: 2079-2084

[20] Carey DL, Blanch P, Ong K-L et al. Training loads and injury risk in Australian football-differing acute: Chronic workload ratios influence match injury risk. Br J Sports Med 2017; 51: 1215-1220

[21] Colby M], Dawson B, Peeling P et al. Multivariate modelling of subjective and objective monitoring data improve the detection of non-contact injury risk in elite Australian footballers. J Sci Med Sport 2017; 20: 1068-1074

[22] Murray NB, Gabbett T], Townshend AD et al. Calculating acute: Chronic workload ratios using exponentially weighted moving averages provides a more sensitive indicator of injury likelihood than rolling averages. Br J Sports Med 2017; 51: 749-754

[23] Rogalski B, Dawson B, Heasman J et al. Training and game loads and injury risk in elite Australian footballers. J Sci Med Sport 2013; 16: 499-503

[24] Ruddy JD, Shield AJ, Maniar N et al. Predictive modeling of hamstring strain injuries in elite Australian footballers. Med Sci Sports Exerc 2018; 50: 906-914

[25] Stares J, Dawson B, Peeling P et al. Identifying high risk loading conditions for in-season injury in elite Australian football players. J Sci Med Sport 2018; 21: 46-51

[26] Esmaeili A, Hopkins WG, Stewart AM et al. The individual and combined effects of multiple factors on the risk of soft tissue non-contact injuries in elite team sport athletes. Front Physiol 2018; 9: 1280

[27] Sampson JA, Murray A, Williams S et al. Injury risk-workload associations in NCAA American college football. J Sci Med Sport 2018 21: $1215-1220$

[28] Sampson JA, Murray A, Williams S et al. Subjective wellness, acute: Chronic workloads, and injury risk in college football. J Strength Cond Res 2019, doi:10.1519/JSC.0000000000003000

[29] Møller M, Nielsen RO, Attermann J et al. Handball load and shoulder injury rate: A 31-week cohort study of 679 elite youth handball players. Br J Sports Med 2017; 51: 231-237

[30] Malone S, Roe M, Doran D et al. High chronic training loads and exposure to bouts of maximal velocity running reduce injury risk in elite Gaelic football. J Sci Med Sport 2017; 20: 250-254

[31] Bowen L, Gross AS, Gimpel M et al. Spikes in acute:chronic workload ratio (ACWR) associated with a 5-7 times greater injury rate in English Premier League football players: A comprehensive 3-year study. $\mathrm{Br}$ ] Sports Med 2019, doi:10.1136/bjsports-2018-099422

[32] Bowen L, Gross AS, Gimpel M et al. Accumulated workloads and the acute: Chronic workload ratio relate to injury risk in elite youth football players. Br J Sports Med 2017; 51: 452-459

[33] Delecroix B, Delaval B, Dawson B et al. Workload and injury incidence in elite football academy players. J Sports Sci 2019; 37: 2768-2773

[34] Fanchini M, Rampinini E, Riggio M et al. Despite association, the acute: Chronic work load ratio does not predict non-contact injury in elite footballers. Sci Med Foot 2018; 2: 108-114

[35] Jaspers A, Kuyvenhoven JP, Staes F et al. Examination of the external and internal load indicators' association with overuse injuries in professional soccer players. J Sci Med Sport 2018; 21: 579-585

[36] Lu D, Howle K, Waterson A et al. Workload profiles prior to injury in professional soccer players. Sci Med Foot 2017; 1: 237-243

[37] McCall A, Dupont G, Ekstrand J. Internal workload and non-contact injury: A one-season study of five teams from the UEFA Elite Club Injury Study. Br J Sports Med 2018; 52: 1517-1522
[38] Lolli L, Batterham AM, Hawkins R et al. Mathematical coupling causes spurious correlation within the conventional acute-to-chronic workload ratio calculations. Br J Sports Med 2017; 53: 921-922

[39] Gabbett T], Hulin B, Blanch P et al. To couple or not to couple? For acute: Chronic workload ratios and injury risk, does it really matter? Int J Sports Med 2019; 40: 597-600

[40] Bahr R, Clarsen B, Ekstrand J. Why we should focus on the burden of injuries and illnesses, not just their incidence. $\mathrm{Br}$ ] Sports Med 2018 ; 52: 1018-1021

[41] Walden M, Krosshaug T, Bjorneboe J et al. Three distinct mechanisms predominate in non-contact anterior cruciate ligament injuries in male professional football players: A systematic video analysis of 39 cases. $\mathrm{Br}$ J Sports Med 2015; 49: 1452-1460

[42] Harriss DJ, Macsween A, Atkinson G. Standards for ethics in sport and exercise science research: 2020 update. Int J Sports Med 2019; 40: 813-817

[43] Malone J], Lovell R, Varley MC et al. Unpacking the black box: Applications and considerations for using gps devices in sport. Int J Sports Physiol Perform 2017; 12: S218-S226

[44] Thornton HR, Nelson AR, Delaney JA et al. Interunit reliability and effect of data-processing methods of global positioning systems. Int J Sports Physiol Perform 2019; 14: 432-438

[45] Roe G, Darrall-Jones ], Black C et al. Validity of 10-HZ GPS and timing gates for assessing maximum velocity in professional rugby union players. Int J Sports Physiol Perform 2017; 12: 836-839

[46] Varley MC, Jaspers A, Helsen WF et al. Methodological considerations when quantifying high-intensity efforts in team sport using global positioning system technology. Int J Sports Physiol Perform 2017; 12: 1059-1068

[47] Mueller-Wohlfahrt H-W, Haensel L, Mithoefer K et al. Terminology and classification of muscle injuries in sport: The Munich consensus statement. Br J Sports Med 2013; 47: 342-350

[48] Williams S, West S, Cross MJ et al. Better way to determine the acute: Chronic workload ratio? Br J Sports Med 2017; 51: 209-210

[49] McCall A, Dupont G, Ekstrand J. Internal workload and non-contact injury: A one-season study of five teams from the UEFA Elite Club Injury Study. Br J Sports Med 2018; 52: 1517-1522

[50] Yu HS, Kim J], Kim HW et al. Impact of mechanical stretch on the cell behaviors of bone and surrounding tissues. J Tissue Eng 2016; 7: $1-24$

[51] Vanrenterghem J, Nedergaard NJ, Robinson MA et al. Training load monitoring in team sports: A novel framework separating physiological and biomechanical load-adaptation pathways. Sports Med 2017; 47: 2135-2142

[52] Schache AG, Dorn TW, Blanch PD et al. Mechanics of the human hamstring muscles during sprinting. Med Sci Sports Exerc 2012; 44: 647-658

[53] Gabbett T]. The training-injury prevention paradox: Should athletes be training smarter and harder? Br J Sports Med 2016; 50: 273-280

[54] Windt ], Ardern CL, Gabbett T] et al. Getting the most out of intensive longitudinal data: A methodological review of workload-injury studies. BMJ Open 2018; 8: e022626

[55] Baumert P, Lake M], Stewart CE et al. Genetic variation and exerciseinduced muscle damage: Implications for athletic performance, injury and ageing. Eur J Appl Physiol 2016; 116: 1595-1625

[56] Williams S, Trewartha G, Kemp SPT et al. The influence of an artificial playing surface on injury risk and perceptions of muscle soreness in elite rugby union. Scand J Med Sci Sports 2016; 26: 101-108

[57] von Rosen P, Frohm A, Kottorp A et al. Too little sleep and an unhealthy diet could increase the risk of sustaining a new injury in adolescent elite athletes. Scand J Med Sci Sports 2017; 27: 1364-1371 
[58] Mangine GT, Hoffman JR, Gonzalez AM et al. Bilateral differences in muscle architecture and increased rate of injury in National Basketball Association players. J Athl Train 2014; 49: 794-799

[59] Padaki AS, Noticewala MS, Levine WN et al. Prevalence of posttraumatic stress disorder symptoms among young athletes after anterior cruciate ligament rupture. Orthop J Sports Med 2018; 6: 2325967118787159
[60] Hagglund M, Walden M, Magnusson $\mathrm{H}$ et al. Injuries affect team performance negatively in professional football: An 11-year follow-up of the UEFA Champions League injury study. Br J Sports Med 2013; 47: 738-742

[61] Nielsen RO, Bertelsen ML, Ramskov D et al. Time-to-event analysis for sports injury research part 1: Time-varying exposures. $\mathrm{Br}$ । Sports Med 2019; 53: 61-68 